\title{
Dispersive power of gratings and prisms
}

Carlos Robledo-Sanchez, Alberto Jaramillo-Nunez, D. Gale, Gilberto Camacho

Carlos I. Robledo-Sanchez, Alberto Jaramillo-Nunez, D. M. Gale, Gilberto Camacho, "Dispersive power of gratings and prisms," Proc. SPIE 3831, Sixth International Conference on Education and Training in Optics and Photonics, (16 June 2000); doi: 10.1117/12.388698

Event: Education and Training in Optics and Photonics (ETOP'99), 1999, Cancun, Mexico 


\title{
Dispersive power of gratings and prisms
}

\author{
C.I. Robledo-Sánchez, A. Jaramillo-Núñez*, D. Gale*, G. Camacho \\ FCFM, BUAP, Apdo. Postal 1152, Puebla, Pue. México, 72000 \\ *INAOE, Apdo. Postal 51 and 216, Puebla, Pue. , México. 72000 \\ E-mail: crobledo@fcfm.buap.mx, ajaramil@inaoep.mx
}

\begin{abstract}
From the general definition of dispersion power, analytic expressions are deduced for the chromatic dispersion of a prism and a diffraction grating. This deduction is routinely presented in an optics postgraduate course, where emphasis is placed on the explanation of the phenomenon of dispersion in two apparently distinct optical elements.
\end{abstract}

Key words: Prisms, gratings, chromatic dispersion.

\section{INTRODUCTION}

Prisms and gratings are two optical elements which produce similar effects. An example is the change in direction of propagation suffered by a wavefront incident on such elements, or the chromatic dispersion effect produced with white light. Importantly, the chromatic dispersion effect finds application in some optical instruments to analyse the spectral composition of light. To measure the degree of dispersivity of an optical element one refers to the dispersive power. The dispersive power $(\gamma)$ is defined in a general sense as the relative deviation of a beam of light from its original trajectory as a function of a wavelength. Mathematically, $\gamma$ is calculated according to

$$
\gamma=\frac{\delta \theta}{\theta}
$$

Applying this definition one thus obtains the dispersive power of a prism or diffraction grating.

1.1 Prims. The deviation suffered by a ray on traversing a prism with apex angle $\alpha$ is given by $\theta=\alpha(n-1)$. The derivative of $\theta$ with respect to $n$ is $\delta \theta=\alpha \delta n$, and substitution of $\alpha$ from the first equation gives

$$
\delta \theta=\theta \frac{\delta n}{n-1}
$$

Hence the dispersive power, from Eq. (1), is simply $\gamma_{p}=\frac{\delta n}{n-1}$. Using the spectral lines $f, c$ and $d$, we can write the dispersive power as

$$
\gamma_{p}=\frac{n_{f}-n_{c}}{n_{d}-1}
$$

1.2 Grating. Applying the equation for dispersive power (1) to the grating equation, we have 


$$
a \theta=m \lambda,
$$

where $a$ is the grating period, $m$ the order of diffraction and $\lambda$ the wavelength. Differenciating with respect to $\theta$ one obtains $\delta \theta=\frac{m \delta \lambda}{a}$, and substitution for $\frac{m}{a}$ in equation (4) gives

$$
\gamma_{g}=\frac{\delta \lambda}{\lambda}
$$

Hence for the spectral lines $f, d$ and $c$, the dispersion of a grating is given by

$$
\gamma_{g}=\frac{\lambda_{f}-\lambda_{c}}{\lambda_{d}} .
$$

Another important parameter related to the dispersive power is the Abbe number $\left(V=\gamma^{-1}\right)$. Thus for a prism

$$
V_{p}=\frac{n_{d}-1}{n_{f}-n_{c}}
$$

and for a grating

$$
V_{g}=\frac{\lambda_{d}}{\lambda_{f}-\lambda_{c}} .
$$

The equations (7) and (8) are deduced from a general definition of dispersion power. The importance of this result is that it is applied for two dispersive elements.

\section{ACKNOWLEDMENTS}

We acknowledge the support of Consejo Nacional de Ciencia y Tecnologia (CONACYT).

\section{REFERENCES}

1. Hecht E., Optics, Addison-Wesley Publishing Co. Chap. 6, Massachusetts, (1987).

2. Sweatt W. C., "Describing holographic optical elements as lenses", JOSA, 67, 803 (1977).

3. Davison A., Friesen A and Hasman, "Analytic design of hibrid diffractive-refractive achromats", Appl. Opt. 32, $4770^{\circ}(1993)$. 